15th International Spin Physics Symposium

(SPIN 2002)

Upton, New York

BNL-71697-2003-CP

September 9-14, 2002

1

\title{
Spin Coupling Resonance and Suppression in the AGS
}

\author{
V. H. Ranjbar, S. Y. Lee* and L. Ahrens, M. Bai, K. Brown, W. Glenn, H. \\ Huang, A. U. Luccio, W. W. MacKay, V. Ptitsyn, T. Roser, N. Tsoupas ${ }^{\dagger}$ \\ "Indiana University Bloomington IN 47405 \\ ${ }^{\dagger}$ BNL, Upton NY 11973
}

\begin{abstract}
Spin depolarizing resonances due to coupling may account for as much as a 30 percent loss in polarization in the AGS. The major source of coupling in the AGS is the solenoidal snake. In the past some preliminary work was done to understand this phenomena [1], and a method to overcome these resonances was attempted [2]. However, in the polarized proton run of 2002, the response of these coupled spin.resonances to the strength of the solenoidal snake, skew quadrupoles and vertical and horizontal betatron tune separation was studied to provided a benchmark for a modified DEPOL program [3]. Then using the new DEPOL program, a method method to cure the coupled spin resonances in the AGS via spin matching rather than global or local decoupling was explored.
\end{abstract}

\section{UPDATE ON MODIFICATION TO DEPOL PROGRAM}

In previous papers [10] we reported on the modifications to the well established DEPOL code [3] to include the effects of coupling. We present now some additional modifications which have significantly improved the speed of this code. The central algorithm presented in [10] is created to evaluate the following Fourier integral,

$$
\varepsilon_{K_{m}}=-\frac{1}{2 \pi} \int_{s_{1}}^{s_{2}}\left[(1+K)\left(z^{\prime \prime}+\frac{i z^{\prime}}{\rho}\right)-i(1+G)\left(\frac{z}{\rho}\right)^{\prime}\right] e^{i K \theta(s)} d s .
$$

Here $\varepsilon_{K}$ is the spin resonance amplitude and $K$ is the spin resonance tune. The solution, following the original DEPOL paper, was to break up the integral into a sum over all the lattice elements denoted with subscript $m$. The final closed solution for each element is:

$$
\begin{array}{r}
\varepsilon_{m}=\frac{1}{2 \pi}\left[\frac{(1+K)\left(\xi_{1}+i\right)}{\rho} z_{1} e^{i K \theta_{1}}+\frac{(1+K)\left(\xi_{2}-i\right)}{\rho} z_{2} e^{i K \theta_{2}}\right. \\
-(1+K)\left(\left(z_{2}^{\prime}-\frac{i K}{\rho} z_{2}\right) e^{i K \theta_{2}}-\left(z_{1}^{\prime}-\frac{i K}{\rho} z_{1}\right) e^{i K \theta_{1}}\right)+\left(\frac{K\left(K^{2}+G\right)}{\rho^{2}}\right)
\end{array}
$$

\footnotetext{
${ }^{1}$ Work performed under the auspices of the US Department of Energy
} 


$$
\begin{array}{r}
\times\left[\frac { 1 } { \sqrt { 1 + | r _ { e } | } } \left(\left(\frac{i K}{\rho} r_{e_{1,2}}-r_{e_{1,1}}\right)\left(\frac{\left(a_{2}^{\prime}-\frac{i K}{\rho} a_{2}\right) e^{i K \theta_{2}}-\left(a_{1}^{\prime}-\frac{i K}{\rho} a_{1}\right) e^{i K \theta_{1}}}{K_{a}-K^{2} / \rho^{2}}\right)\right.\right. \\
\left.\left.-\left(\frac{\left(b_{2}^{\prime}-\frac{i K}{\rho} b_{2}\right) e^{i K \theta_{2}}-\left(b_{1}^{\prime}-\frac{i K}{\rho} b_{1}\right) e^{i K \theta_{1}}}{K_{b}-K^{2} / \rho^{2}}\right)+r_{e_{1,2}}\left(a_{2} e^{i K \theta_{2}}-a_{1} e^{i K \theta_{1}}\right)\right)\right]
\end{array} .
$$

Here $r_{e}$ is the rotation matrix which transforms from the $x, x^{\prime}, z, z^{\prime}$ coupled basis to the $a, a^{\prime}, b, b^{\prime}$ locally uncoupled basis (uncoupling each lattice element only). Since for intrinsic resonances $K$ is not an integer Eq. ?? becomes an integral around the lattice an infinite number of times. Previously, a solution was derived by evaluating an appropriately large number of passes over the lattice.

However if we look closely at the behavior of the elements which make up the integral to be evaluated in Eq. 2 it appears that we can factor out the phase element which changes with each period around the lattice. The remaining elements in the sum remain constant for each pass. The factored plase elements can be evaluated analytically using the properties of a geometric series. The results are four separate enhancement functions,

$$
\begin{aligned}
& E_{u}(N)_{ \pm}=\sum_{n=0}^{N} e^{i 2 \pi n\left(K \pm v_{u}\right)}= \pm e^{i N \pi\left(K \pm v_{u}\right)} \frac{\sin \left(\pi(N+1)\left(K \pm v_{u}\right)\right)}{\sin \left(\pi\left(K \pm v_{u}\right)\right)} \\
& E_{v}(N)_{ \pm}=\sum_{n=0}^{N} e^{i 2 \pi n\left(K \pm v_{v}\right)}= \pm e^{i N \pi\left(K \pm v_{v}\right)} \frac{\sin \left(\pi(N+1)\left(K \pm v_{v}\right)\right)}{\sin \left(\pi\left(K \pm v_{v}\right)\right)}
\end{aligned}
$$

Here $v_{u}$ and $v_{v}$ the betatron tunes in the uncoupled $u$ and $v$ basis. $\mathrm{N}$ the number of passes around the lattice. The function once evaluated can then be multiplied by the appropriate terms in the sum over one pass in the lattice.

\section{STUDY OF COUPLING SPIN RESONANCES}

The primary source of coupling in the AGS is the partial solenoidal snake. In addition there exists a family of six skew quadrupoles. It has been observed that the bare AGS machine has a net skew quadrupole moment. Coupling studies in the past estimated the average roll to be $0.13 \mathrm{mrad}$ [9]. Additionally closed orbit errors can contribute to coupling via feed down from the sextupole fields present in the AGS combined function magnets and sextupole magnets.

During the 2002 polarized proton run, particular attention was paid to studying the behavior of the $0+v_{x}$ resonance crossing since the analyzing power of the AGS polarimeter was sufficiently large at low energy to generate accurate measurements and the strength of the $0+v_{x}$ coupled spin resonance was large. Initial DEPOL calculations without rolls generated curves which were too broad. It was only by including either a large single roll or selectively placed rolls that a good fit to the measured data was achieved. For all DEPOL calculations shown here selectively distributed rolls were ap- 
plied to the CF magnets ${ }^{2}$ ( 0.05 mrad per magnet) and applied to the BD magnets ${ }^{3}$ ( $0.25 \mathrm{mrad}$ per magnet ). This is not unreasonable considering previous estimates. However it should be emphasized that this configuration is by no means unique. While it was essential to include a net skew quadrupole moment in the bare AGS the distribution and the direction of these rolls is still unclear since our data could fit many different configurations.

In Figs. 1 - 3 one can see the results of our tune scans, snake scans and skew quadrupole scans. All calculations assume a $70 \%$ polarization at injection into the AGS. In all cases we were able to achieve a good agreement between DEPOL polarization and measured.

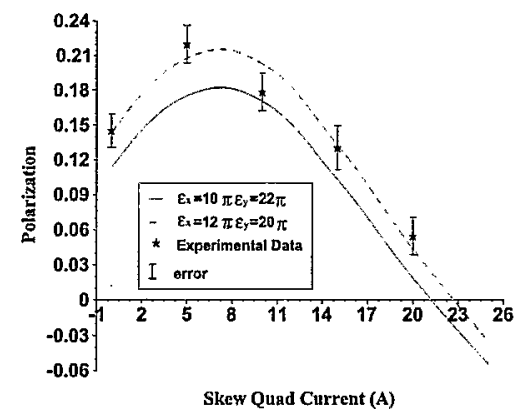

FIGURE 1. Polarization after crossing the $0+v_{x}$ and $0+v_{z}$ resonances with set vertical tune and horizontal tunes $\left(v_{z}=8.8, v_{x}=8.78\right)$. Scanning through skew quadrupole input currents from 0 to 25 A. The vertical and horizontal emittances were measured as $(11 \pm 1) \pi$ and $(21 \pm 1) \pi$ mm-mrad.

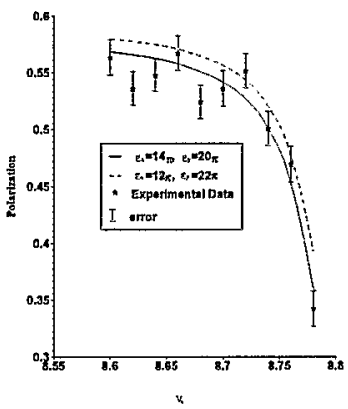

FIGURE 2. Polarization after crossing the $0+v_{x}$ and $0+v_{z}$ resonances with set vertical tune $\left(v_{z}=\right.$ 8.8) scanning horizontal set tunes. Vertical and horizontal emittances were measured as $(13 \pm 1) \pi$ and $(21 \pm 1) \pi$ mm-mmrad respectively.

${ }^{2} \mathrm{CF}$ is the label for a family of combined function focusing magnets located at $13,14,17$ and 18 positions in each super-period.

${ }^{3} \mathrm{BD}$ is the label for a family of combined function focusing magnets located at $11,12,19$ and 20 positions in each super-period. 


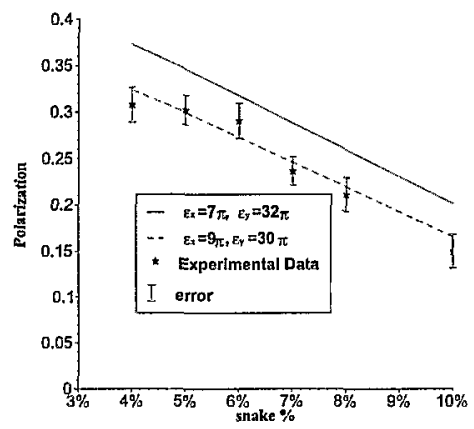

FIGURE 3. Polarization after crossing the $0+v_{x}$ and $0+v_{z}$ resonances with fixed vertical tune and horizontal tune $\left(v_{z}=8.8, v_{x}=8.7\right)$ scanning from 4 to $10 \%$ partial snake strength. Vertical and horizontal emittances were measured to be $(8 \pm 1) \pi$ and $(30 \pm 1) \pi \mathrm{mm}$-mrad.

\section{SUPPRESSION OF THE COUPLED SPIN RESONANCES}

In the AGS, six skew quadrupoles are located in the 17th location at every other super period. To globally decouple the AGS, ideally one should pick a location with as large a phase difference from the existing skew quadrupoles. Unfortunately, we are limited in the number of free locations. The 15th location which has been suggested for the future normal quadrupole could also accommodate a skew quadrupole.

Unfortunately, the field strength required to approach a situation of global decoupling cause a large tune shifts in the AGS which makes identifying the proper strengths necessary to decouple the machine difficult if not impossible. However, since we are concerned with eliminating the coupled spin resonances and not necessarily decoupling the AGS, a spin matching condition may still exist. Using spin matching it may be possible to either partly or totally cancel the coupled spin resonance with the perturbation introduced by the skew quadrupoles. In the plots in Fig. 4 such a spin matching condition appears achievable. For these figures we have fixed our vertical and horizontal tunes ( $v_{z}=8.8, v_{x}=8.7$ ) and scanned through various current strengths for the 15th and 17th skew quadrupoles. For these calculations the 15 th skew quadrupoles were assumed to have the same size and current to field transfer function as the existing skew quadrupoles in the 17th lattice postion. For all four resonances a solution appears possible. However, overcoming the $36+v_{x}$ requires a current in excess of $1200 \mathrm{~A}$. Since this high current needs to be maintained only during the brief milliseconds of the resonance crossing it should be possible to achieve.

Actually since the calculations where all done using the slower acceleration rate of $\alpha=2.4 \times 10^{-5}$ generated by the backup Westinghouse power generator and not the usual $\alpha=4.8 \times 10^{-5}$ which is normally achieved by the Siemens power generator, depolarization could effectively be overcome with a stronger residual resonance. 

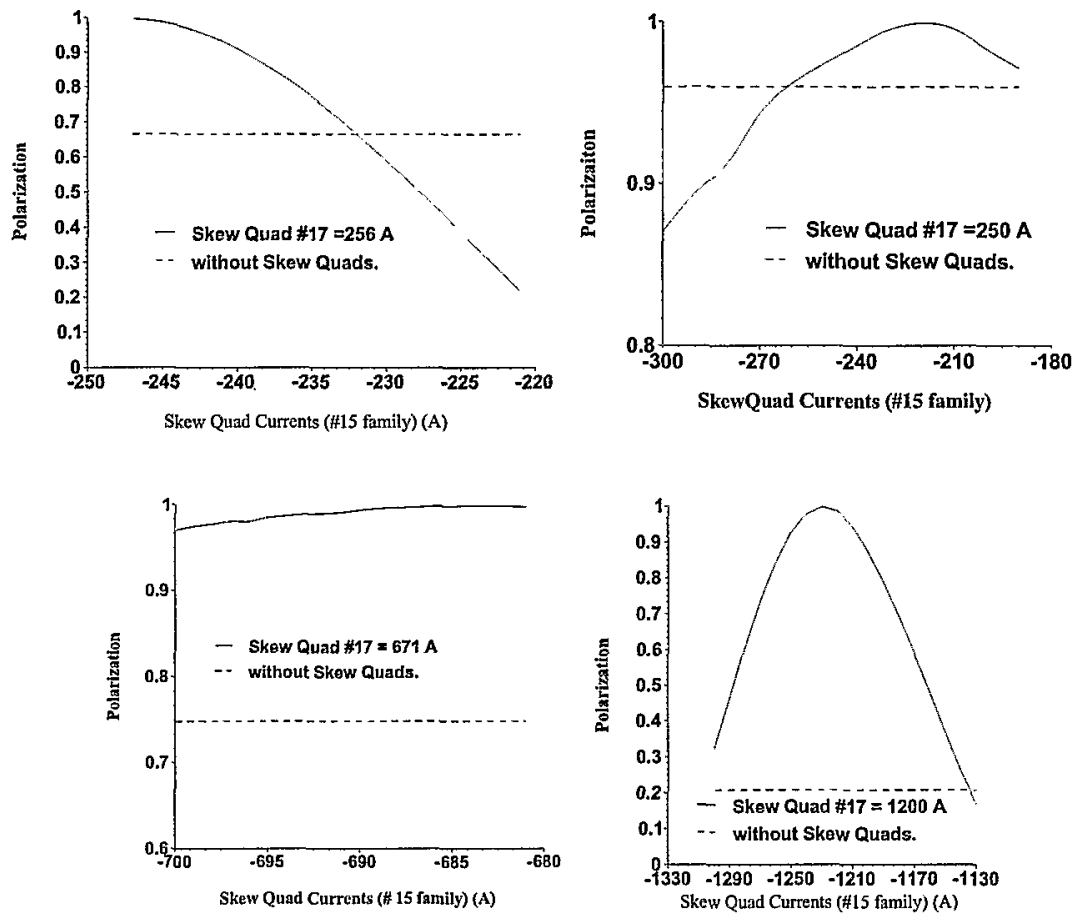

FIGURE 4. Polarization after crossing (clockwise) $0+v_{x}, 12+v_{x}, 36-v_{x}$, and $36+v_{x}$ resonance with fixed vertical tune and horizontal tune $\left(v_{z}=8.8, v_{x}=8.7\right)$. Scanning currents for a hypothetical skew quadrupole in the 15 th lattice position and the 17 th skew quadrupole family at fixed.

\section{REFERENCES}

1. H. Huang, Ph. D Thesis, Indiana University (1995)

2. M. Bai and T. Roser. C-A/AP/37 (2001).

3. E. D. Courant and R. D. Ruth, BNL Report 51270 (1980).

4. H.Huang et al., Phys. Rev. Lett. 73, 2982 (1994).

5. M.Bai et al, Phys. Rev E56, 6002 (1997).

6. L. C. Teng, FN 229, FNAL Report, (1971).

7. H.Grote and F.C.Iselin, Methodical Accelerator Design Program Version 8.23, CERN/SL/90-13(AP) (1990).

8. A. Zelenski, et al., Proceedings of the 9th International Conference on Ion Sources, Rev. Sci.Inst., Vol. 73, No.2, p.888 (2002).

9. C.J.Gardner, et. al, AGS Studies Report, N224, (1987).

10. V.Ranjbar et al., Mapping out the full spin resonance structure of RHIC, PAC 2001 Proc.

11. J. Y. Lin, Phys. Rev. E 49, 2347 (1994). 